\title{
Quality Map Generation in Two-Dimensional Phase Unwrapping Process by Using Edge Detection Techniques
}

\author{
Yuangang Lu, Wancheng Zhao and Xuping Zhang \\ Nanjing University \\ China
}

\section{Introduction}

Two-dimensional phase unwrapping is the task of recovering the true phase values, given the wrapped phase values in an image. Phase unwrapping arises in several branches of applied optics, physics, medicine and engineering, such as homomorphic signal processing, solid-state physics, optical interferometry, adaptive or compensated optics, magnetic resonance imaging, synthetic aperture radar interferometry, and optical and electron holography (Volkov \& Zhu, 2003). In these applications, the measured information is denoted by a two-dimensional phase distribution called the wrapped phase image. In wrapped phase images, the phase is the interval $(-\Pi, \Pi]$ or $(0,2 \Pi]$ due to the use of the mathematical arctangent function (Bone, 1991). Since this wrapped phase suffers from 2-ח phase jumps, it is unusable until the phase discontinuities are removed. In order to recovering the true continuous phase values to denote real physical quantity, a phase unwrapping process is needed to recovering the true phase values. The procedure of phase unwrapping is performed by either adding or subtracting integer multiples of $2 \Pi$ to all successive pixels when a phase discontinuity encounters, which are based on some kind of threshold mechanism (Ghiglia et al., 1987).

However, many factors, such as surface discontinuities, noise, under-sampling, or shadow, would produce unreliable phase data, which make the recovery of the wrapped phase challenging. To solve the problem, many phase unwrapping algorithms have been developed during the last three decades. These phase unwrapping algorithms can be found in a very good reference book (Ghigli \& Pritt, 1998) and review papers such as (Baldi et al., 2002; Jenkinson, 2003; Su \& Chen, 2004; Zappa \& Busca, 2008). In many phase unwrapping algorithms, a quality map, which evaluated the quality or the reliability of the phase data, is used for completing the phase unwrapping process. In wrapped phase images, the quality of a pixel is low if it is located in areas where the surface discontinuities, noise or undersampling exists. On the contrary, the quality of a pixel is high if it is located in areas where the variation of phase value is low. From the mathematical point of view, quality map is a matrix of the same size of phase image that assign a quality value to each pixel. The quality values are usually normalized in the range $[0,1]$, where a large value means high reliability of the corresponding pixel. In most phase unwrapping algorithms, a quality map is necessary to guide the phase unwrapping process for achieving desire results. Furthermore, 
whether the unwrapping result is satisfied, to a large extent, depends on whether the quality map can truly reflect the phase data quality.

Until now there are more than ten quality maps proposed to guide the two-dimensional phase unwrapping. As we will see, most quality maps are based on the phase gradients or phase derivatives of the wrapped phase images, and thus can be obtained by edge detection techniques. It is well known that gradient estimation is the first step in the widely used three-step edge detection procedure (Meer \& Georgescu, 2001). Therefore, edge detection techniques, which aim at identifying points at which the image brightness changes sharply, play important roles in two-dimensional phase unwrapping process. By means of edge detection techniques, new quality maps based on weighted phase gradients can be generated to effectively guide phase unwrapping process.

This chapter will review the existing literature related to quality map, enlarge the family of quality map by using edge detection techniques. In the following Section 2 , the existing quality maps will be reviewed, and the performance of some widely used quality maps on phase unwrapping will be evaluated by unwrapping three typical intractable wrapped phase images. Then, in Section 3, some new quality maps, which are based on existing methods, will be developed from a perspective of edge detection techniques. Finally, conclusions are drawn and directions for further research on quality maps are outlined in Section 4.

\section{Existing quality maps}

In this section we review existing quality maps that are used in phase unwrapping process. Some quality maps widely used in phase unwrapping process will be evaluated by unwrapping three typical intractable wrapped phase images.

Most quality maps can be directly derived from wrapped phase image that is generated from the original measurement data, but there are some kinds of quality maps that can only be derived from the original measurement data, such as intensity of fringe pattern. According to their origin, quality maps can be divided into two categories: quality map derived from wrapped phase image and quality map derived from original measurement data.

\subsection{Quality map derived from wrapped phase image}

The wrapped phase images can be obtained from most of the phase measurements such as magnetic resonance imaging, synthetic aperture radar, adaptive optics, and optical interferometry. Fig. 1 shows three examples of the wrapped phase images. The first one is obtained by computer simulation and its corresponding real phase image is shown in Fig. 1 (a), which is $256 \times 256$ pixels in size. The image is defined by two spiral plane surfaces that have been tilted relative to one another (Ghigli \& Pritt, 1998). The wrapped phase image of the Fig. 1(a) is shown in Fig. 1(b), where the phase of each pixel is wrapped into the interval $(-\Pi, \Pi]$. In the figure, wrapped phase image is scaled between black and white to cover the full dynamic range. The real phase discontinuities between the two spiral surfaces make the phase unwrapping intractable. Fig. 1(c) shows the wrapped phase image of an optical fiber connector endface, which is also $256 \times 256$ pixels in size. It is the measurement data by use of four step phase shifted optical interferometry (Kwon, 1984). Unlike the phase data obtained by computer simulation, the original real phase data cannot be given because it is unknown before the wrapped image is unwrapped. As we can see, the wrapped phase image is corrupted by salt-and-pepper noise, especially in the center of image. In addition, in this area the dense fringe may cause under-sampling problem. The last example shown in Fig. 
$1(\mathrm{~d})$ is a curved surface with a rectangle hole obtained by computer simulation, and the wrapped phase image of Fig. 1(d) is shown in Fig. 1(e). The wrapped image is $100 \times 100$ pixels in size. It is suffered from the surface discontinuities and shadow in a closed rectangle hole below the center of the image, and the phase along the vertical direction of image changes more complicated than that of image shown in Fig. 1(b). These factors make its phase unwrapping difficult.

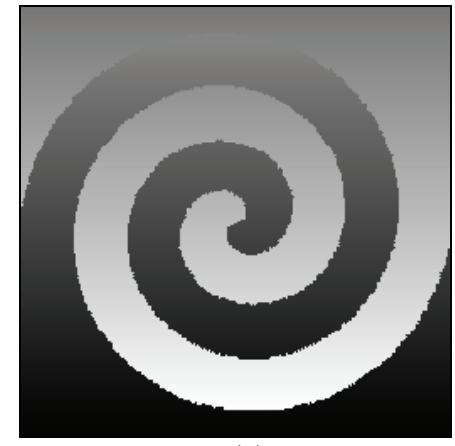

(a)

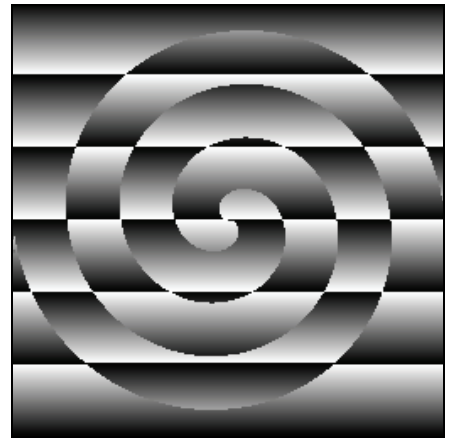

(b)

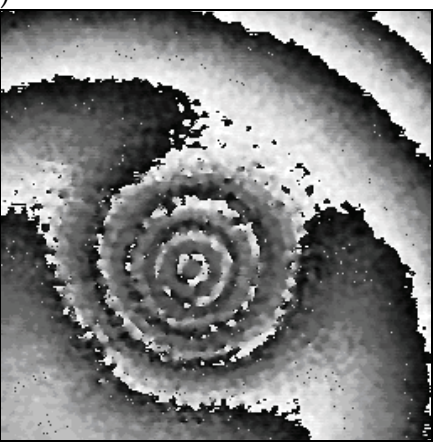

(c)

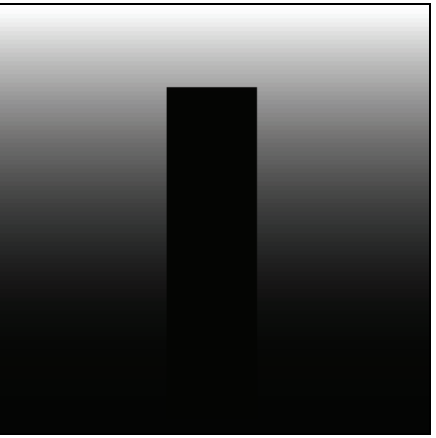

(d)

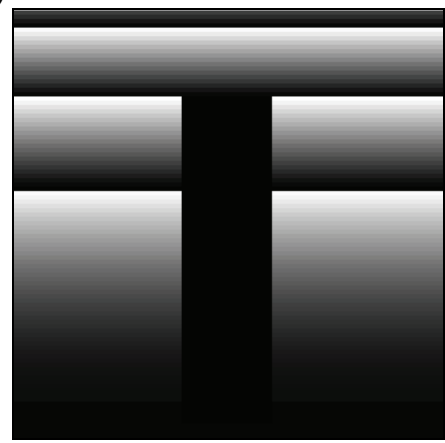

(e)

Fig. 1. (a) The spiral shear surface. (b) The wrapped phase image of Fig. 1(a). (c) The wrapped phase image of the optical fiber connector endface. (d) The curved surface with a rectangle hole. (e) The wrapped phase image of figure 1(d). 
In this and the following sections, the three wrapped phase images will be unwrapped by a quality guide phase unwrapping algorithm (Flynn, 1996) using some quality maps that can be derived from the wrapped phase images.

\subsubsection{Pseudo-correlation map}

The pseudo-correlation map (Ghiglia \& Pritt, 1998) is designed to measure the correlation of the wrapped phase images. The value of the quality map for pixel $(m, n)$ is calculated according to

$$
q_{m, n}=\frac{\sqrt{\left(\sum \cos \psi_{i, j}\right)^{2}+\left(\sum \sin \psi_{i, j}\right)^{2}}}{k^{2}},
$$

where $\psi_{i, j}$ is the wrapped phase value and the sums are evaluated in the $\mathrm{k} \times \mathrm{k}$ neighborhood centered at each pixel $(\mathrm{m}, \mathrm{n})$. Normally, $\mathrm{k}$ is 3 or 5 in practice.

The pseudo-correlation map is based on the correlation of the wrapped phase image. It is sensitive to the noisy phase data, since the noisy regions of wrapped phase image are normally the low correlation regions. However, the pseudo-correlation map may mark the reliable regions with steep slopes as low quality regions. This is due to the low correlation of phase data in these regions. In this sense, a conservative strategy is chosen in this quality map. It is unreasonable to presume that all the low correlation areas should be marked as low quality. Therefore, the unwrapping results obtained by use of pseudo-correlation map are not always correct, especially when the wrapped phase image contains reliable regions with steep slopes.

The pseudo-correlation maps of the three wrapped phase images are shown in Fig. 2(a), 2(c) and 2(e), respectively. $3 \times 3$ window is chosen to compute the correlation of wrapped phase images. The values of quality maps are normalized into $[0,1]$ and the low quality values are shown as dark pixels and the high quality values as light pixels. Fig. 2(b), 2(d) and 2(f), respectively, show the unwrapping results by use of quality guide algorithm and the pseudo-correlation maps shown respectively in Fig. 2(a), 2(c) and 2(e). The result of the optical fiber connector endface, which shown in Fig. 2(d), is satisfactory. The pseudocorrelation map copes well with the intractable wrapped phase image, producing a correct unwrapping result. However, the other two unwrapping results, Fig. 2(b) and 2(f), are incorrect. The pseudo-correlation map incorrectly marks the steep surfaces of the spiral shear and curved surface, which are shown in Fig. 1(a) and 1(d) respectively, as low quality regions. Furthermore, and more importantly, the map fails to isolate the sheared planes from one another in spiral shear surface, and fails to isolate the closed area from other areas in the curved surface. These factors result in some degree of phase-unwrapping error. So we can say that the pseudo-correlation map is not a very good estimator of phase quality.

\subsubsection{Phase derivatives variance map}

The phase derivatives variance map (Ghiglia \& Pritt, 1998) measures the statistical variance of the phase derivatives. The value of this map for pixel $(m, n)$ is expressed as

$$
q_{m, n}=\frac{\sqrt{\sum\left(\Delta_{i, j}^{x}-\overline{\Delta_{m, n}^{x}}\right)^{2}}+\sqrt{\sum\left(\Delta_{i, j}^{y}-\overline{\Delta_{m, n}^{y}}\right)^{2}}}{k^{2}},
$$




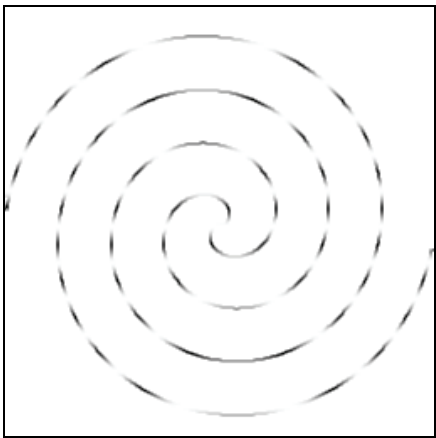

(a)

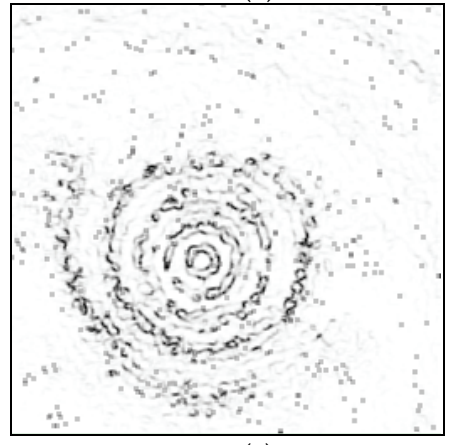

(c)

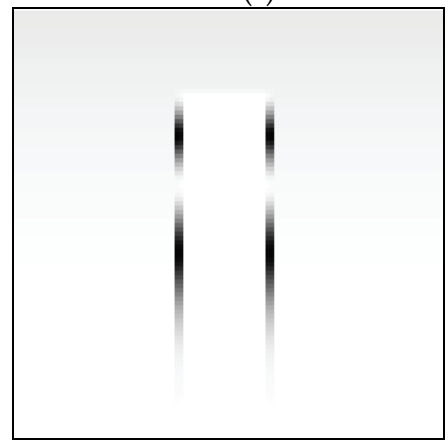

(e)

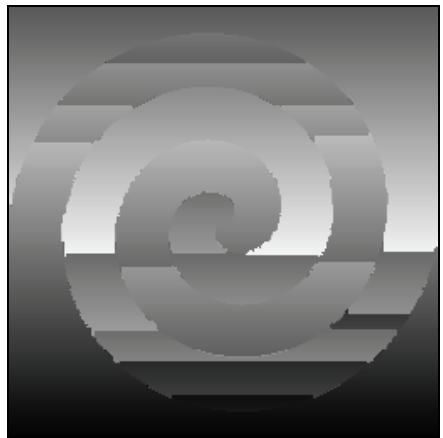

(b)

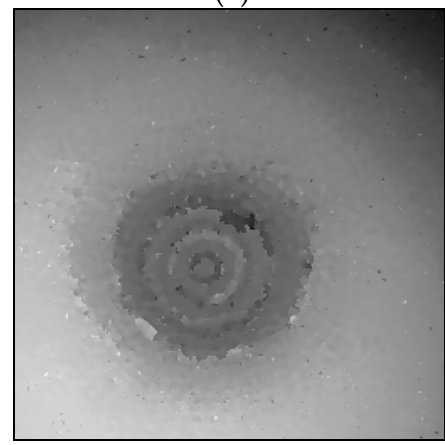

(d)

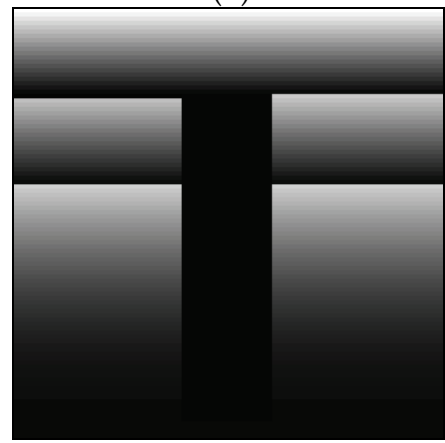

(f)

Fig. 2. (a) Pseudo-correlation map of Fig. 1(b). (b) Unwrapping result by use of Fig. 2(a). (c) Pseudo-correlation map of Fig. 1(c). (d) Unwrapping result by use of Fig. 2(c). (e) Pseudocorrelation map of Fig. 1(e). (f) Unwrapping result by use of Fig. 2(e).

where the terms $\Delta_{i, j}^{x}$ and $\Delta_{i, j}^{y}$ are the partial derivatives of the phase, the terms $\overline{\Delta_{m, n}^{x}}$ and $\overline{\Delta_{m, n}^{y}}$ are the averages of these partial derivatives in the $\mathrm{k} \times \mathrm{k}$ windows, and the sums evaluated in the $\mathrm{k} \times \mathrm{k}$ neighborhood centered at the pixel $(\mathrm{m}, \mathrm{n})$.

The phase derivatives variance map can be estimated as the local sample variance of the phase derivatives. The map indicates the badness of the phase data. In other words, the 
more unreliable the phase data is, the higher the phase derivatives variance is. So it should be negated to represent goodness of the phase data. Unlike the conservative strategy used in the pseudo-correlation map, an aggressive strategy is adopted in the phase derivatives variance map. For example, in regions where the isolated noise points are located, the phase variation is large, but the phase derivatives variance is low. Therefore, the phase derivatives variance map may mark the noisy regions as high quality area, especially when the size of window $\mathrm{k}$ is large.

The phase derivatives variance map is based on the phase derivatives of the wrapped phase image. However, if the phase gradients or derivatives are used directly to the construction of quality map, that will result in error quality map. Taking the wrapped spiral shear surface for example, its phase derivatives variance map is shown in Fig. 3. As it can be seen, the $2 \Pi$ phase jumps lines, i.e. the horizontal lines, in the wrapped phase are detected and marked as low quality. Actually, the phase jump lines are not unreliable regions, so the quality map shown in Fig. 3 does not reflect the real quality of the wrapped phase, which will result in an incorrect unwrapping result.

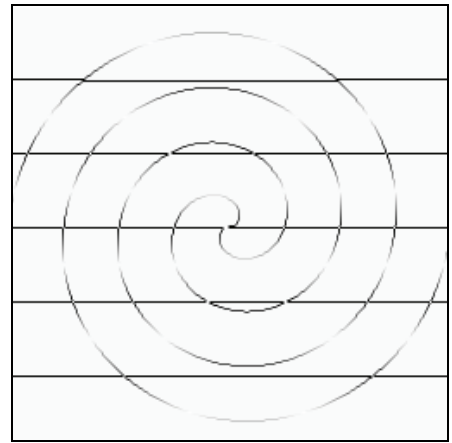

Fig. 3. Phase derivatives variance map of wrapped spiral shear surface without removing $2 \Pi$ phase jumps.

Therefore, before phase derivatives are obtained, a pre-processing is needed to remove the $2 \Pi$ phase jump lines. When the phase jump lines are detected, $2 \Pi$ is added or subtracted to remove the phase jumps. Then the phase derivatives can be extracted, and thus the quality map can be obtained. For all quality maps based on the phase derivatives, the preprocessing is need to avoid the influence of $2 \pi$ phase jumps

The phase derivatives variance maps of the three wrapped phase images are shown in Fig. $4(\mathrm{a}), 4(\mathrm{c})$ and $4(\mathrm{e})$, respectively. A $3 \times 3$ window is chosen to compute the variance of the phase derivatives. These quality maps are different from the pseudo-correlation maps shown in Fig. 2(a), 2(c), and 2(e). For example, where the phase exhibits a constant variation, such as that induced by the spiral shear surface, the phase derivative variance is zero while the pseudo-correlation is nonzero. The variance operation to phase gradient makes the quality map more reliable.

Fig. 4(b), 4(d) and 4(f) respectively, show the unwrapping results by means of quality guide algorithm and the phase derivatives variance maps shown respectively in Fig. 4(a), 4(c) and 4(e). The unwrapping result of the spiral shear surface shown in Fig. 4(b) is just the same as the phase data shown in Fig. 1(a). The unwrapping result is quite good. The unwrapping result of the optical fiber connector endface shown in Fig. 4(d) is defective since there is 
some mistake in the center of the map. This may result from the salt- and-pepper noise. Fig. $4(f)$ shows the unwrapping result of the curved surface wrapped phase. The result is also unsatisfactory, since the quality map fails to strictly differentiate the reliable and unreliable areas due to the presence of the real phase discontinuities and shadow.

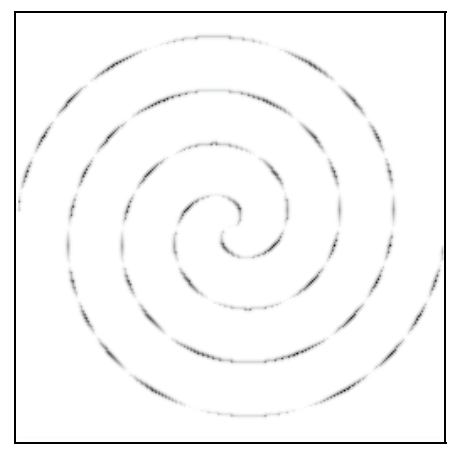

(a)

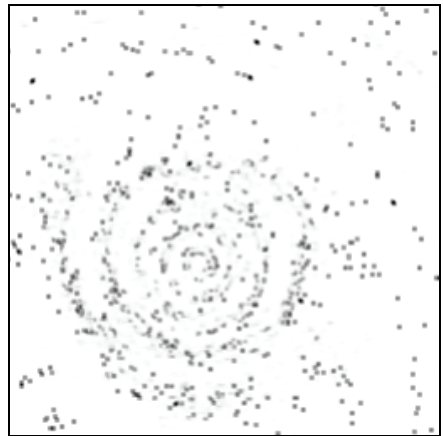

(c)

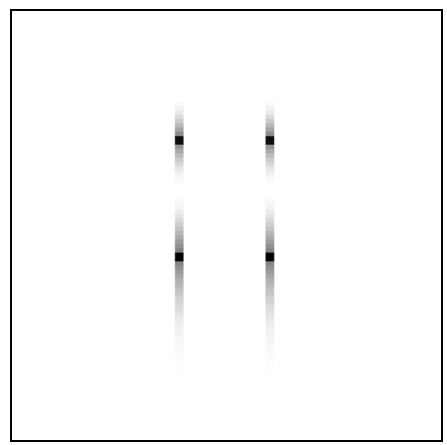

(e)

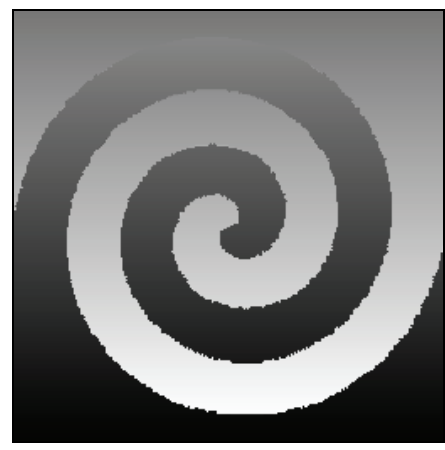

(b)

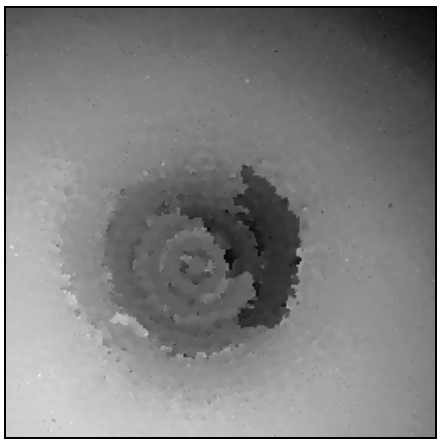

(d)

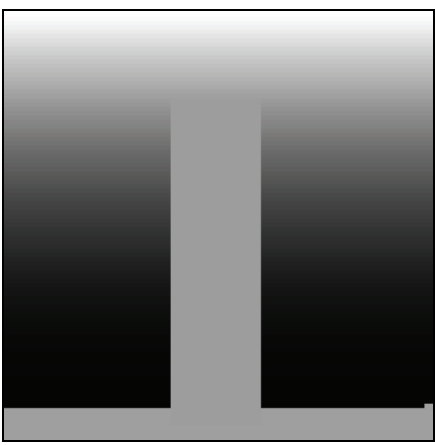

(f)

Fig. 4. (a) Phase derivatives variance map of Fig. 1(b). (b) Unwrapping result by use of Fig. 4(a). (c) Phase derivatives variance map of Fig. 1(c). (d) Unwrapping result by use of Fig. 4(c). (e) Phase derivatives variance map of Fig. 1(e). (f). Unwrapping result by use of Fig. 4(e). 


\subsubsection{Maximum phase gradient map}

The maximum phase gradient map (Ghiglia \& Pritt, 1998) is defined as the largest phase gradient value in the $\mathrm{k} \times \mathrm{k}$ neighborhood of each pixel. The value of the quality map for pixel $(\mathrm{m}, \mathrm{n})$ is calculated according to

$$
z_{m, n}=\max \left\{\max \left\{\left|\Delta_{i, j}^{x}\right|\right\}, \max \left\{\left|\Delta_{i, j}^{y}\right|\right\}\right\},
$$

where the terms $\Delta_{i, j}^{x}$ and $\Delta_{i, j}^{y}$ are the partial derivatives of the phase and the max are evaluated in $\mathrm{k} \times \mathrm{k}$ neighborhoods of the given pixel.

The maximum phase gradient map takes the largest phase gradient value in the $k \times k$ neighborhood of each pixel as quality value. Therefore, it also denotes the badness rather than the goodness of the phase data, just as the phase derivatives variance map. The disadvantage of the maximum phase gradient map is that the reliable regions with steep slopes will be masked as low quality, which is similar to that of pseudo-correlation map. However, the map has an advantage that it is sensitive to the noisy phase data. In the regions where the noisy phase is present, the gradients tend to be large and thus the quality values are low.

The maximum phase gradient map of the three wrapped phase data are shown respectively in Fig. 5(a), 5(c) and 5(e). A $3 \times 3$ window is chosen to compute the maximum phase gradient. Fig. 5(b), 5(d) and 5(f) respectively show unwrapping results by use of quality guide algorithm and the maximum phase gradient maps shown respectively in Fig. 5(a), 5(c) and 5(e). Because the quality maps fail to mark the quality of reliable regions with steep slopes as high values, the unwrapping results of the spiral shear surface and curved phase are defective, as that shown in Fig. 5(b) and 5(f). The unwrapping result of the optical fiber connector endface shown in Fig. 5(d) is correct, due to the maximum phase gradient map's high sensitivity on the noisy phase area.

\subsubsection{Derivative variance correlation map}

The derivative variance correlation map (Lu et al., 2005; Lu et al., 2006; Lu et al., 2007) is a hybrid from two commonly used quality maps, the pseudo-correlation map and the derivative variance map. The value of the quality map for pixel $(m, n)$ is calculated according to

$$
q_{m, n}=\frac{\sqrt{\sum\left(\Delta_{i, j}^{x}-\overline{\Delta_{m, n}^{x}}\right)^{2}}+\sqrt{\sum\left(\Delta_{i, j}^{y}-\overline{\Delta_{m, n}^{y}}\right)^{2}}}{k^{2}},
$$

where $\psi_{i, j}$ is the wrapped phase values, the terms $\Delta_{i, j}^{x}$ and $\Delta_{i, j}^{y}$ are the partial derivatives of the phase, the term $\overline{\Delta_{m, n}^{x}}$ and $\overline{\Delta_{m, n}^{y}}$ are the averages of these partial derivatives in the $\mathrm{k} \times \mathrm{k}$ windows, and the sums evaluated in the $\mathrm{k} \times \mathrm{k}$ neighborhood centered at each pixel $(\mathrm{m}, \mathrm{n})$. Normally $\mathrm{k}$ is 3 or 5 in practice.

Because the derivative variance correlation map is a hybrid from the pseudo-correlation map and the derivative variance map, it has advantages of both of the latter two maps. For example, it is sensitive to the noisy phase data like the pseudo-correlation map, and works well in steep areas. 


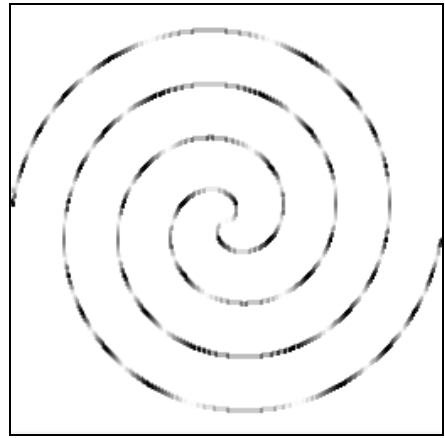

(a)

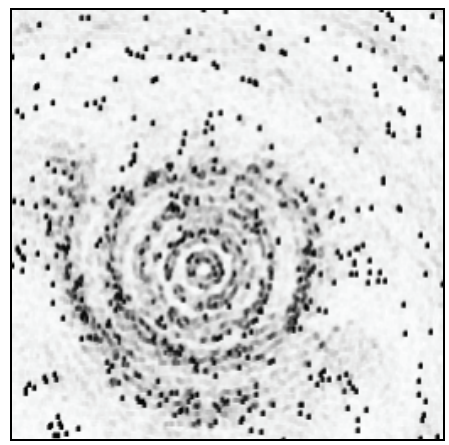

(c)

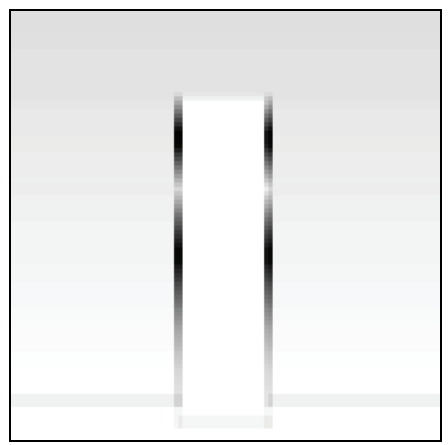

(e)

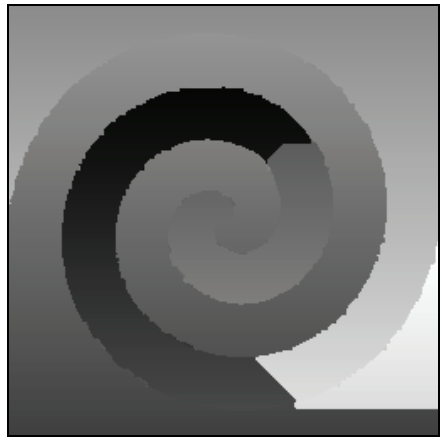

(b)

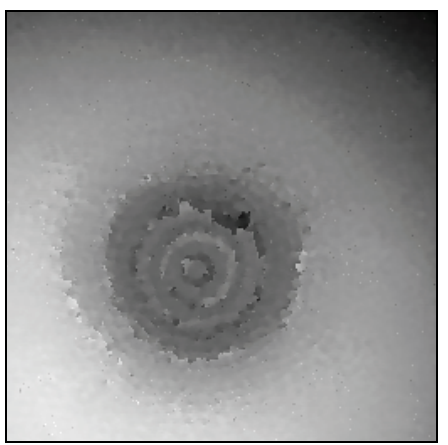

(d)

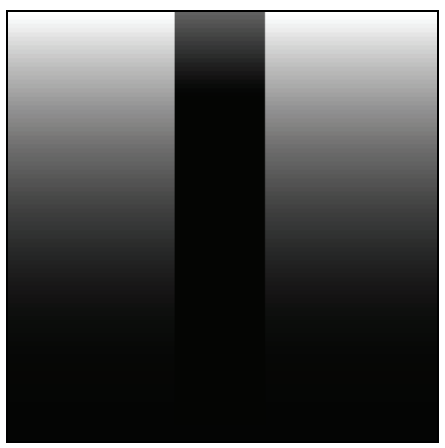

(f)

Fig. 5. (a) Maximum phase gradient map of Fig. 1(b). (b) Unwrapping result by use of Fig. 5(a). (c) Maximum phase gradient map of Fig. 1(c). (d) Unwrapping result by use of Fig. 5 (c). (e) Maximum phase gradient map of Fig. 1(e). (f) Unwrapping result by use of Fig. 5 (e).

The derivative variance correlation maps of the three wrapped phase data are shown in Fig. 6(a), 6(c) and 6(e), respectively. A $3 \times 3$ window is chosen to compute the value of quality map. Fig. 6(b), 6(d) and 6(f) respectively show the unwrapping results by means of quality guide algorithm and the derivative variance correlation maps shown respectively in Fig. 
6(a), 6(c) and 6(e). The unwrapping results are satisfactory. So we can see that the map can truly reflect wrapped phase quality, ensuring a more reliable unwrapping result. However, it is more complicated than some other maps.

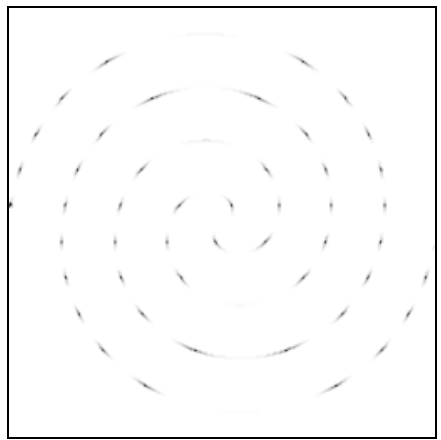

(a)

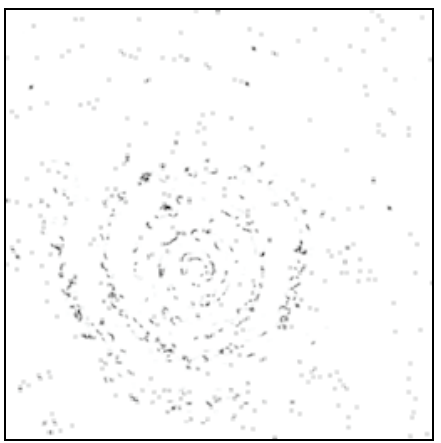

(c)

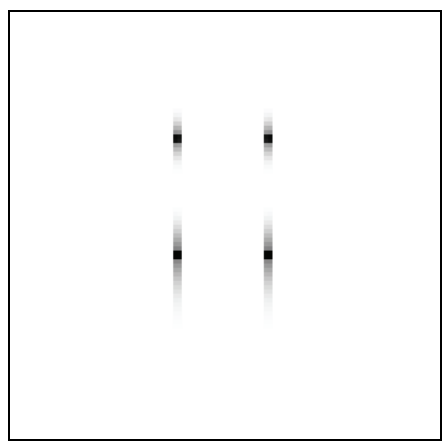

(a)

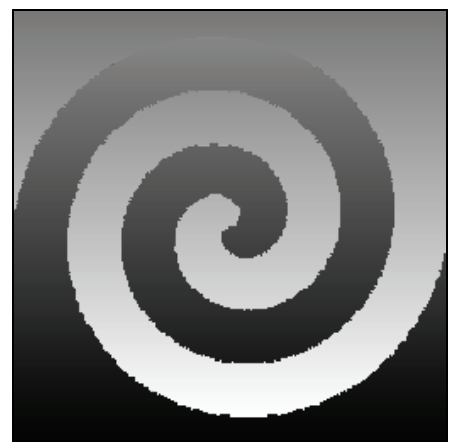

(b)

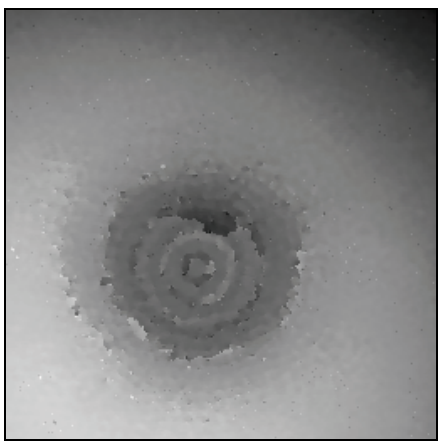

(d)

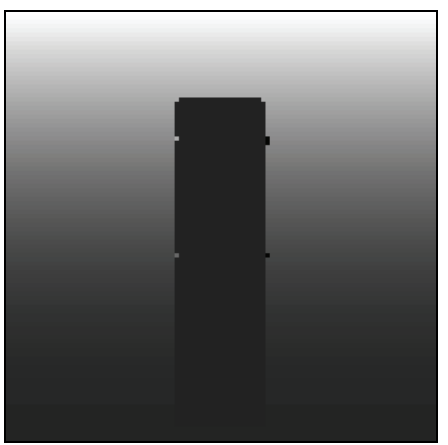

(b)

Fig. 6. (a) Derivative variance correlation map of Fig. 1(b). (b) Unwrapping result by use of Fig. 6(a). (c) Derivative variance correlation map of Fig. 1(c). (d) Unwrapping result by use of Fig. 6(c). (e) Derivative variance correlation map of Fig. 1(e). (f). Unwrapping result by use of Fig. 6(e). 


\subsubsection{Some other quality maps}

There are also a few quality maps derived from wrapped phase data other than the quality maps mentioned above. The residue-vector map (Karout et al., 2007) is a quality map which combines both the concept of the quality maps and the knowledge of the residues and their branch cuts. The windowed Fourier filtering quality map (Qian et al., 2008) is based on the filtered amplitude derived by the windowed Fourier filtering. There are also hybrid quality maps which are combination of the existing two maps. For example, the quality map combined by pseudo-correlation map and maximum phase gradient map (Lu et al., 2004), and the second differences of the phase data can also be used to construct quality maps (Bone, 1991).

\subsection{Quality maps derived from original measurement data}

\subsubsection{Correlation map}

The correlation map (Ghiglia \& Pritt, 1998) is defined by the correlation coefficients of the interferometric synthetic aperture radar (IFSAR) data. The value of the quality map for pixel $(\mathrm{m}, \mathrm{n})$ is calculated according to

$$
q_{m, n}=\frac{\sum u_{i, j} \overline{v_{i, j}}}{\sqrt{\sum\left|u_{i, j}\right|^{2} \sum\left|v_{i, j}\right|^{2}}}
$$

where $\overline{v_{i, j}}$ is the complex conjugate of $v_{i, j}, u_{i, j}$ and $v_{i, j}$ are the corresponding complex values from the two images after interpolation. The sum is performed in the $\mathrm{k} \times \mathrm{k}$ neighborhood centered at each pixel $(m, n)$.

The correlation map is the best estimator of the quality of the phase data extracted from the IFSAR. However, it cannot be derived from the wrapped phase data, which is the most commonly used phase data in the phase unwrapping algorithms.

\subsubsection{Fringe modulation map}

The fringe modulation map is based on the intensity modulation of the fringe pattern, which is the original measurement data in optical interferometry. Therefore, the quality map can be extracted from the phase shifted interferogram. The value of the fringe modulation quality map for pixel $(\mathrm{m}, \mathrm{n})$ is calculated according to

$$
q_{m, n}=\sqrt{\left(\sum_{i=1}^{N} I_{i}(m, n) \sin (2 \pi n / N)\right)^{2}+\left(\sum_{i=1}^{N} I_{i}(m, n) \cos (2 \pi n / N)\right)^{2}},
$$

where $\mathrm{N}$ is the number of phase shifts employed in the measurement processing, and $\mathrm{I}_{\mathrm{i}}$ is the intensity distribution of the $i$ th phase shifting fringe pattern.

The fringe modulation map can be derived by the phase shifted interferogram or a single fringe pattern from which the wrapped phase can be obtained. In phase-shift interferometry, at least 3 fringe patterns are needed to obtain the quality map. That is to say, in equation (6) $\mathrm{N}$ is required no less than 3 . In this case, the corresponding quality map is called as the temporal fringe modulation map (Su et al., 1993). However, when the fringe pattern projected on an object surface is sinusoidal, a quality map can be derived from a single 
fringe pattern. The value of the quality can be obtained by using equation (6) where $\mathrm{N}$ equals 1. Accordingly, the quality map is named as the spatial fringe modulation map (Quan et al., 2003).

The fringe modulation map is regarded as a kind of reliable and robust map. However, like the correlation map, it cannot be directly extracted from the wrapped phase data. Therefore, it cannot be used to guide phase unwrapping when the original measurement data are not available.

\section{Quality maps derived by the edge detecting techniques}

As we can see, several kinds of quality maps are based on the phase derivatives, such as the phase derivative variance map, the maximum phase gradient map and the derivative variance correlation map. The phase derivatives reflect the phase qualities to some extent and play important roles in the construction of quality maps. As that mentioned in section 1 , the phase derivatives can be obtained by edge detection techniques. Some new quality maps based on weighted phase gradients can be generated by means of edge detection techniques. Strictly speaking, the process of obtaining the quality map based on phase derivatives or gradients in section 2 also contains the edge detection techniques. The edge detection masks are the first order phase derivatives, which are shown in Fig. 7. They are the simplest masks in edge detection techniques. In this figure, " $X$ " denotes the horizontal direction and " $Y$ " the vertical direction, and the underlined elements indicates the origin of the mask. Beyond that, there are some other commonly used edge detection masks(Gonzalez, 2002). Masks of size $2 \times 2$ are shown in Fig. 8(a), which are very simple masks in edge detection named as Roberts

$$
\begin{aligned}
& \left(\begin{array}{ll}
\frac{-1}{0} & 1 \\
0 & 0
\end{array}\right)\left(\begin{array}{ll}
\frac{-1}{1} & 0 \\
1 & 0
\end{array}\right) \\
& \begin{array}{ll}
X & Y
\end{array}
\end{aligned}
$$

Fig. 7. The masks of first-order differential operator. The underlined elements in the two masks indicate the location of origin.

$$
\begin{array}{r}
\left(\begin{array}{ll}
\frac{-1}{0} & 0 \\
0 & 1
\end{array}\right)\left(\begin{array}{cc}
0 & -1 \\
1 & 0
\end{array}\right) \\
X \underset{Y}{\mathrm{Y}}\left(\begin{array}{ccc}
-1 & -1 & -1 \\
0 & 0 & 0 \\
1 & 1 & 1
\end{array}\right)\left(\begin{array}{ccc}
-1 & 0 & 1 \\
-1 & 0 & 1 \\
-1 & 0 & 1
\end{array}\right) \\
\text { X }
\end{array}
$$

(a)

(b)

(c)

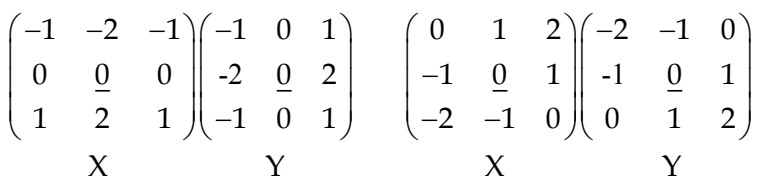

(e)

Fig. 8. (a) The masks of Roberts operators. (b) The masks of Prewitt operators. (c) The masks of modified Prewitt operators. (d) The masks of Sobel operators. (e) The masks of modified Sobel operators. The underlined elements in these masks indicate the location of origin. 
operators. The masks of Prewitt operators and its modified form are shown in Fig. 8(b) and Fig. 8(c), respectively. The Prewitt operators have the strongest responses along the Fig. 8(c), respectively. The Prewitt operators have the strongest responses along the horizontal and vertical direction, but the modified Prewitt operators are more suitable for detecting diagonal edges. The masks of Sobel operators and its modified form are shown in Fig. 8(d) and Fig. 8(e), respectively. Because the masks shown in Fig. 8 can be seen as the weighted forms of the masks shown in Fig. 7, new weighted quality maps can be developed by means of these edge detection operators. In this section, two weighted quality maps derived by the edge detecting techniques will be presented.

\subsection{Weighted phase derivatives variance maps}

Weighted phase derivatives variance maps can be generated from the equation (2), in which the value of $\Delta_{i, j}^{x}$ and $\Delta_{i, j}^{y}$ and is obtained by use of the operators shown in Fig. 8 . As that mentioned in section 2, a pre-processing is needed to remove the $2 \Pi$ phase jump lines before the weighted versions of $\Delta_{i, j}^{x}$ and $\Delta_{i, j}^{y}$ can be obtained.

The masks of Prewitt operators are chosen to generate the weighted phase derivatives variance map. The quality maps of the wrapped phase images shown in Fig. 1(b), 1(c) and 1(e), are shown in Fig. 9(a), 9(c) and 9(e), respectively. The corresponding unwrapping result by use of the quality guide algorithm and the weighted phase derivatives variance map, are shown in Fig. 9(b), 9(d) and 9(f) respectively. The unwrapping results of the optical fiber connector endface and the curved surface is quite right though there may be a little defective in some regions. The unwrapping result of the spiral shear surface is also correct, although it looks different from the original phase data shown in Fig. 1(a). This is because that the two surfaces of the spiral shear surface, which are tilted relative to one another, are unwrapped separately in the process of phase unwrapping. There may be $2 \mathrm{kn}$ phase difference between the two unwrapped surfaces, but it can be eliminated by add the known phase difference to one of the unwrapped surface. In Fig. 9(b), the two surfaces are both unwrapped correctly, so it can be thought as a good unwrapping result. When compare the results with the unwrapping results shown in Fig. 4(b), 4(d) and 4(f), we can find that the results of the spiral shear surface are correct, while the results of the optical fiber connector endface and the curved phase in Fig. 9 are much better than that shown in Fig. 4(d) and 4(f). Therefore, suitable masks of edge detection operators can be utilized to improve the quality of the unwrapping results.

\subsection{Weighted maximum phase gradient map}

Another new quality map generated by means of edge detection techniques is the weighted maximum phase gradient map. Like the phase derivatives variance map, the maximum phase gradient map is based on the phase derivatives. Thus the edge detection techniques can be utilized to enlarge its family. Weighted maximum phase gradient maps can be generated from the equation (3), in which the value of $\Delta_{i, j}^{x}$ and $\Delta_{i, j}^{y}$ is obtained by use of the operators shown in Fig. 8. A pre-processing is also needed to remove the $2 \pi$ phase jump lines before the weighted versions of $\Delta_{i, j}^{x}$ and $\Delta_{i, j}^{y}$ can be obtained. 


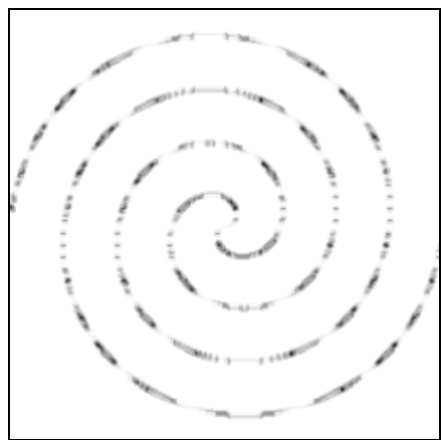

(a)

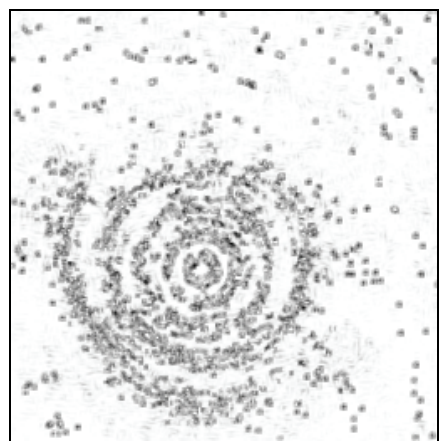

(c)

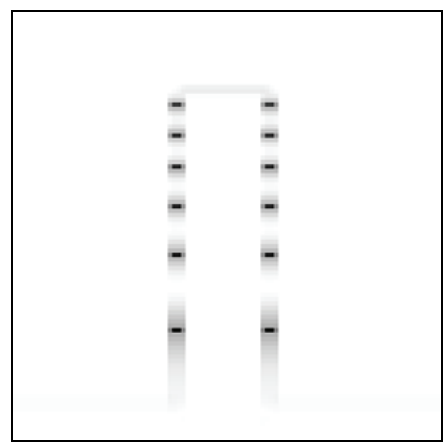

(e)

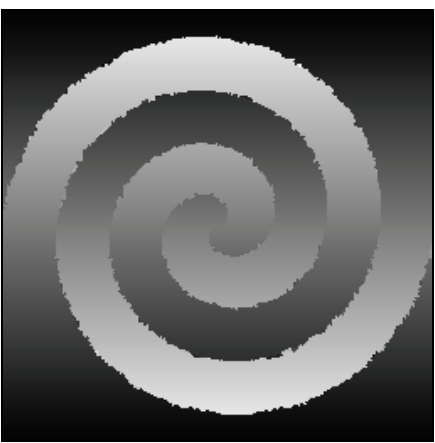

(b)

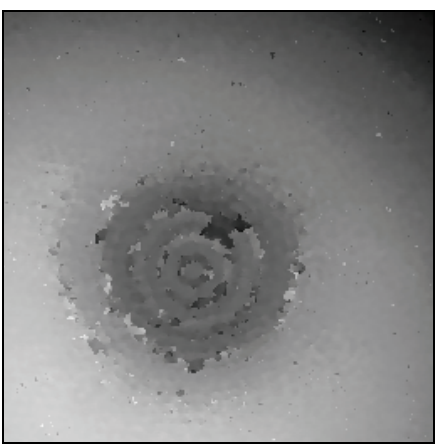

(d)

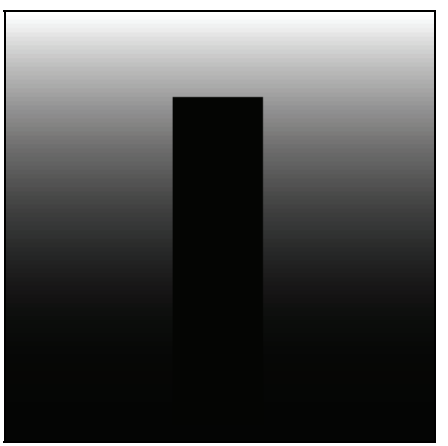

(f)

Fig. 9. (a) Weighted phase derivatives variance map of Fig. 1(b) generated by use of Prewitt operators. (b) Unwrapping result by use of Fig. 9(a). (c) Weighted phase derivatives variance map of Fig. 1(c) generated by use of Prewitt operators. (d) Unwrapping result by use of Fig. 9 (c). (e) Weighted phase derivatives variance map of Fig. 1(e) generated by use of Prewitt operators. (f) Unwrapping result by use of Fig. 9(e). 


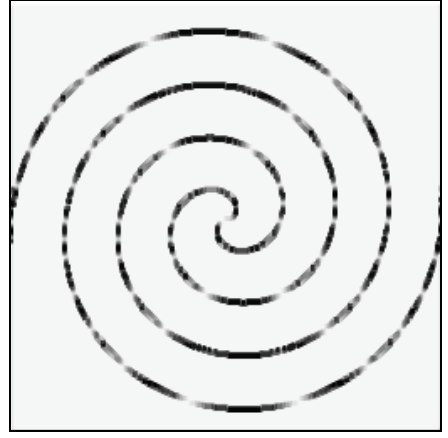

(a)

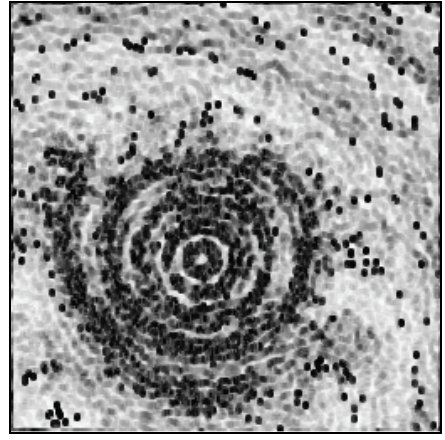

(c)

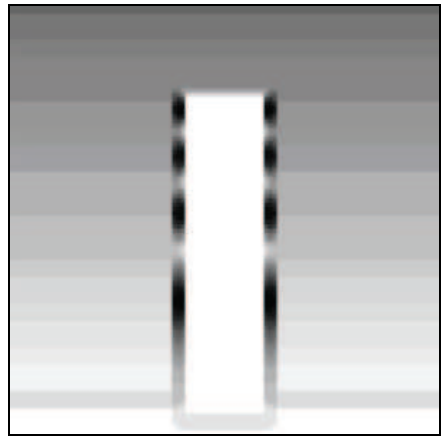

(e)

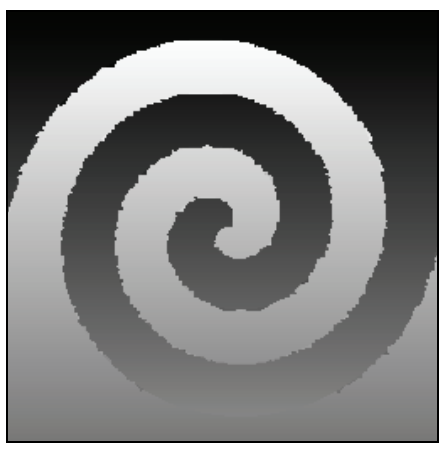

(b)

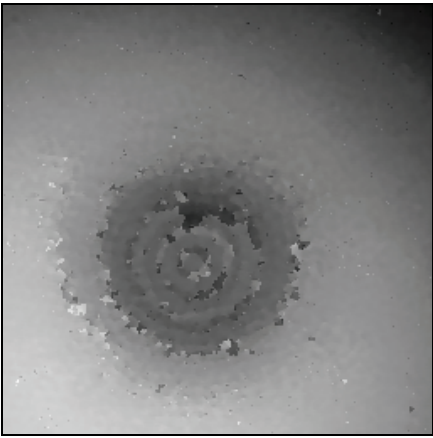

(d)

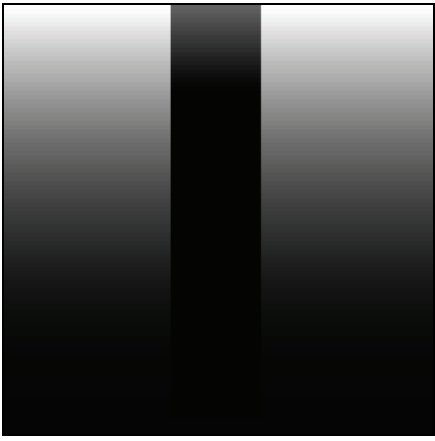

(f)

Fig. 10. (a) The weighted maximum phase gradient map of Fig. 1(b) generated by use of diagonal versions of Prewitt operators. (b) Unwrapping result by use of Fig. 10(a). (c) The weighted maximum phase gradient map of Fig. 1(c) generated by use of diagonal versions of Prewitt operators. (d) Unwrapping result by use of Fig. 10(c). (e) The weighted maximum phase gradient map of Fig. 1(e) generated by use of diagonal versions of Prewitt operators. (f). Unwrapping result by use of Fig. 10(e). 
In this section, the masks in Fig. 8(c), i.e. the diagonal version of Prewitt operators, are chosen. The weighted maximum phase gradient map of the three wrapped phase data are shown in Fig. 10(a), 10(c) and 10(e), respectively. Fig. 10(b), 10(d) and 10(f) show the unwrapping results by use of quality guide algorithm and the weighted maximum phase gradient maps shown respectively in Fig. 10(a), 10(c) and 10(e). Compare the unwrapping results with that shown in Fig. 5(b), 5(d) and 5(f) respectively. The unwrapping results of the optical fiber connector endface and the curved phase are just the same as that shown in Fig. 5(d) and 5(f), where the result of curved surface is defective. The unwrapping result of the spiral shear surface is quite good and much better than the result shown in Fig. 5(b), though there exist $2 \mathrm{k} \Pi$ phase difference between the two surfaces, which makes the unwrapping result looks different from the original phase data as that shown in Fig. 9(b). Therefore, because the diagonal versions of Prewitt operators have strong responses along the diagonal directions, the operators are the suitable masks that can be used in the case of the spiral shear surface unwrapping.

\section{Conclusion and future work}

Quality maps play very important role in two-dimensional phase unwrapping process. In this chapter we have introduced the quality map generation method using the edge detection techniques. Existing quality maps which are commonly used in phase unwrapping process have been reviewed, and we find that each quality map has its advantages and disadvantages. Edge detection techniques are utilized to enlarge the family of quality maps due to the fact that most quality maps are based on the phase gradients or phase derivatives of the wrapped phase images. Some new quality maps, which are weighted versions of existing quality maps, are developed by means of edge detection operators. The unwrapping results show that appropriate edge detection operator can make the quality map more reliable.

Although more than ten quality maps have been proposed and we enlarge their family by constructing a few of new quality maps in this work, there are still some challenges related to the quality maps in future work. For example, although we can use edge detection techniques to obtain a new quality map, we still need to evaluate its reliability. Until now, there are no recognized standards in this important area. The common evaluation method is evaluating the unwrapping results of some kinds of intractable wrapped phase images. However, the wrapped phase images are chosen arbitrarily and the visual evaluation of unwrapping results may not be accurate. Therefore, the standard wrapped phase images should be identified for evaluating the reliability of quality map, and the uniform standard should be developed for judging the reliability of quality map. Besides, new quality maps based on new features of the wrapped phase image, such as phase correlation or the entropy of phase data, should be developed to make up for the deficiencies of the existing quality maps.

\section{Acknowledgments}

It is gratefully noted that the work was supported by the National Natural Science Foundation of China under Grant No. 60607007. 


\section{References}

Baldi, A.; Bertolino, F. \& Ginesu, F. (2002). On the performance of some unwrapping algorithms, Opt Laser Eng, Vol. 37, pp. 313-330

Bone, D. J. (1991). Fourier fringe analysis: the two-dimensional phase unwrapping problem, Appl. Opt, Vol. 30, pp. 3627-3632

Flynn, T. J. (1996). Consistent 2-D phase unwrapping guided by a quality map, Proceedings of the 1996 International Geoscience and Remote Sensing Symposium, pp. 2057-2059, Lincoln, NE, May 1996

Ghiglia, D.; Mastin, G. \& Romero, L. (1987). Cellular-automata method for phase unwrapping, J. Opt. Soc. Am.A, Vol. 4, No. 1, pp. 267- 280

Ghiglia, D. C. \& Pritt, M. (1998). Two-dimensional phase unwrapping: theory, algorithms, and software, Wiley-Interscience publication, New York

Gonzalez, R. C. \& Woods, R. E. (2002). Digital Image Processing Second Edition, Pearson Education, Inc., Prentice Hall

Jenkinson, M. (2003). Fast, automated, N-dimensional phase-unwrapping algorithm, Magnetic Resonance in Medicine, Vol. 49, pp. 93-197

Karout, S. A.; Gdeisat, M. A.; Burton, D. R. \& Lalor, M. J. (2007). Residue vector, an approach to branch-cut placement in phase unwrapping: theoretical study. Appl. Opt., Vol. 46, No. 21, pp. 4712-4727

Kwon, O. Y. (1984). Multichannel phase-shifted interferometer, Opt. Lett., Vol. 9, No. 2, pp. 59-61

Lu, Y.; Wang, X.; Zhong, X.; He, G.; Liu, Y. \& Zheng, D. (2004). A new quality map for quality-guided phase unwrapping, Chinese optics letters, Vol. 2, No. 12, pp. 698700

Lu, Y.; Wang, X. \& He, G. (2005). Phase unwrapping based on branch cut placing and reliability ordering, Optical Engineering, Vol. 44, No. 5, 055601

Lu, Y. \& Zhang, X. (2006). Minimum $\mathrm{L}^{0}$-norm two-dimensional phase unwrapping algorithm Based on the derivative variance correlation map, Journal of Physics: Conference Series, Vol. 48, pp. 308-312

Lu, Y.; Wang, X. \& Zhang, X. (2007). Weighted least-squares phase unwrapping algorithm based on derivative variance correlation map, Optik, Vol. 118, pp. 62-66

Meer, P. \& Georgescu, B. (2001). Edge detection with embedded confidence, IEEE Transactions on pattern analysis and machine intelligence, Vol. 23, No. 12, pp. 13511365

Qian, K.; Gao, W. \& Wang, H. (2008). Windowed Fourier-filtered and quality-guided phaseunwrapping algorithm, Applied optics, Vol. 47, No. 29, pp. 5420-5428

Quan, C.; Tay, C. J.; Chen, L. \& Fu, Y. (2003). Spatial-fringe-modulation-based quality map for phase unwrapping, Appl. Opt.Vol. 42, No. 35, pp. 7060-7065

$\mathrm{Su}$, X.; von Bally, G. \& Vukicevic, D. (1993). Phase-stepping grating profilometry: utilization of intensity modulation analysis in complex object evaluation, Opt. Comm., Vol. 98, pp. 141-150

Su, X. \& Chen, W. (2004). Reliability-guided phase unwrapping algorithm: a review, Opt. Lasers Eng, Vol. 42, pp. 245-261 
Volkov, V. V. \& Zhu, Y. (2003). Deterministic phase unwrapping in the presence of noise, Opt. Lett, Vol. 28, No. 22, pp. 2156-2158

Zappa, E. \& Busca, G. (2008). Comparison of eight unwrapping algorithms applied to Fourier transform profilometry, Opt. Lasers Eng, Vol. 46, pp. 106-116 


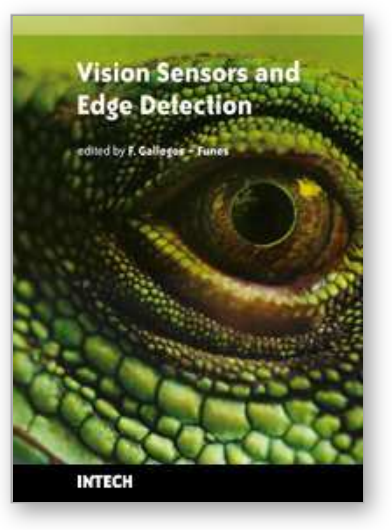

\author{
Vision Sensors and Edge Detection \\ Edited by Francisco Gallegos-Funes
}

ISBN 978-953-307-098-8

Hard cover, 196 pages

Publisher Sciyo

Published online 12, August, 2010

Published in print edition August, 2010

Vision Sensors and Edge Detection book reflects a selection of recent developments within the area of vision sensors and edge detection. There are two sections in this book. The first section presents vision sensors with applications to panoramic vision sensors, wireless vision sensors, and automated vision sensor inspection, and the second one shows image processing techniques, such as, image measurements, image transformations, filtering, and parallel computing.

\title{
How to reference
}

In order to correctly reference this scholarly work, feel free to copy and paste the following:

Yuangang Lu, Wancheng Zhao and Xuping Zhang (2010). Quality Map Generation in Two-Dimensional Phase Unwrapping Process by Using Edge Detection Techniques, Vision Sensors and Edge Detection, Francisco Gallegos-Funes (Ed.), ISBN: 978-953-307-098-8, InTech, Available from:

http://www.intechopen.com/books/vision-sensors-and-edge-detection/quality-map-generation-in-twodimensional-phase-unwrapping-process-by-using-edge-detection-technique

\section{INTECH}

open science | open minds

\section{InTech Europe}

University Campus STeP Ri

Slavka Krautzeka 83/A

51000 Rijeka, Croatia

Phone: +385 (51) 770447

Fax: +385 (51) 686166

www.intechopen.com

\section{InTech China}

Unit 405, Office Block, Hotel Equatorial Shanghai

No.65, Yan An Road (West), Shanghai, 200040, China

中国上海市延安西路65号上海国际贵都大饭店办公楼405单元

Phone: +86-21-62489820

Fax: $+86-21-62489821$ 
(C) 2010 The Author(s). Licensee IntechOpen. This chapter is distributed under the terms of the Creative Commons Attribution-NonCommercialShareAlike-3.0 License, which permits use, distribution and reproduction for non-commercial purposes, provided the original is properly cited and derivative works building on this content are distributed under the same license. 